\title{
Gentamicin induces functional type VII collagen in recessive dystrophic epidermolysis bullosa patients
}

\author{
David T. Woodley, ${ }^{1}$ Jon Cogan, ${ }^{1}$ Yingping Hou, ${ }^{1}$ Chao Lyu, ${ }^{1}$ M. Peter Marinkovich, ${ }^{2,3}$ Douglas Keene, ${ }^{4}$ and Mei Chen ${ }^{1}$ \\ 'Department of Dermatology, The Keck School of Medicine, University of Southern California (USC), Los Angeles, California, USA. ²Department of Dermatology, Stanford University School of Medicine, \\ Stanford, California, USA. ${ }^{3}$ Dermatology, Veteran's Affairs Medical Center, Palo Alto, California, USA. ${ }^{4}$ Shriners Hospital for Children, Portland, Oregon, USA.
}

\begin{abstract}
BACKGROUND. Recessive dystrophic epidermolysis bullosa (RDEB) is an incurable disease caused by mutations in the gene encoding type VII collagen, the major component of anchoring fibrils (AF). We previously demonstrated that gentamicin produced functional type VII collagen in RDEB cells harboring nonsense mutations. Herein, we determined whether topical or intradermal gentamicin administration induces type VII collagen and AFs in RDEB patients.
\end{abstract}

\begin{abstract}
METHODS. A double-blind, placebo-controlled pilot trial assessed safety and efficacy of topical and intradermal gentamicin in 5 RDEB patients with nonsense mutations. The topical arm tested $0.1 \%$ gentamicin ointment or placebo application 3 times daily at 2 open erosion sites for 2 weeks. The intradermal arm tested daily intradermal injection of gentamicin solution ( $8 \mathrm{mg}$ ) or placebo into $\mathbf{2}$ intact skin sites for $\mathbf{2}$ days in $\mathbf{4}$ of $\mathbf{5}$ patients. Primary outcomes were induction of type VII collagen and AFs at the test sites and safety assessment. A secondary outcome assessed wound closure of topically treated erosions.

RESULTS. Both topical and intradermal gentamicin administration induced type VII collagen and AFs at the dermal-epidermal junction of treatment sites. Newly created type VII collagen varied from $20 \%$ to $165 \%$ of that expressed in normal human skin and persisted for 3 months. Topical gentamicin corrected dermal-epidermal separation, improved wound closure, and reduced blister formation. There were no untoward side effects from gentamicin treatments. Type VII collagen induction did not generate anti-type VII collagen autoantibodies in patients' blood or skin.
\end{abstract}

CONCLUSION. Topical and intradermal gentamicin suppresses nonsense mutations and induces type VII collagen and AFs in RDEB patients. Gentamicin therapy may provide a readily available treatment for RDEB patients with nonsense mutations.

TRIAL REGISTRATION. ClinicalTrials.gov NCT02698735.

FUNDING. Epidermolysis Bullosa Research Partnership, Epidermolysis Bullosa Medical Research Foundation, NIH, and VA Merit Award.

\section{Introduction}

Recessive dystrophic epidermolysis bullosa (RDEB) is an incurable, inherited skin disease characterized by skin fragility, blisters, erosions, and scarring (1). RDEB is caused by mutations in the COL7A1 gene that encodes type VII collagen, the major component of anchoring fibrils (AFs) that tether together the 2 main layers of skin, the epidermis and dermis $(2,3)$. With insufficient type VII collagen and AFs, the adherence between the epidermis and dermis is compromised and subepidermal blistering from minor trauma ensues. RDEB families spend large sums of money on health care, with the annual costs readily in excess of $\$ 100,000$ per year. Most patients die in childhood or

Role of funding source: The study funders had no role in the design and conduct of the study, collection, management, analysis, and interpretation of the data; preparation, review, or approval of the manuscript; or decision to submit the manuscript for publication.

Conflict of interest: The authors have declared that no conflict of interest exists.

Submitted: January 17, 2017; Accepted: May 16, 2017

Reference information: / Clin Invest. 2017;127(8):3028-3038.

https://doi.org/10.1172/JCI92707. early adulthood from an aggressive squamous cell carcinoma that develops in one of their chronic skin wounds (4).

The current mainstays of treatment for RDEB are palliative interventions that primarily consist of bandaging the skin, antibiotic treatment of skin infections, recurrent surgical debridement, endoscopic esophageal dilations, periodic transfusions of blood or iron, and nutritional sustenance (5). Unfortunately, there are no cures or even effective treatments for RDEB. Various therapeutic strategies have been envisioned for RDEB, including recombinant type VII collagen-based protein therapy (6-10), bone marrow stem cells, or allogeneic dermal fibroblast-based cell therapy and transplantation of gene-corrected keratinocyte autografts (11-17). However, none of these therapies are consistently effective, and some have associated morbidity and mortality.

According to the Human Gene Mutation Database, $12 \%$ of all mutations reported are nonsense mutations that result in premature termination codons (PTCs) (18). A nonsense mutationsuppression therapy for genetic diseases has emerged based on the ability of aminoglycosides and other compounds to allow translation to proceed through PTCs caused by nonsense mutations $(19,20)$. Aminoglycoside antibiotics, such as gentamicin, 
RDEB patients with bona fide nonsense mutations invited to study $n=5$

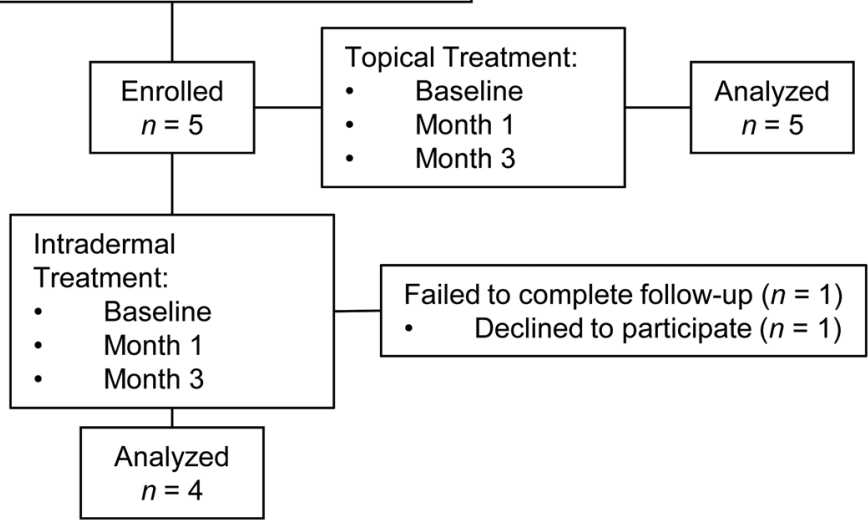

Figure 1. CONSORT flow diagram. Flowchart summarizing RDEB patient enrollment and completion of the trial.

can promote PTC read-through by binding to a specific site in mammalian ribosomal RNA, impairing codon-anticodon recognition at the aminoacyl-transfer RNA acceptor site, and restoring the full-length functional protein $(19,20)$. Aminoglycosides affect PTCs while not appearing to induce significant misreading at normal stop codons in eukaryotes. This is likely due to contextual differences of the mRNAs between normal stop codons and PTCs as well as PTC-associated mRNA retention in the nucleus, which maintains gene expression fidelity $(21,22)$. Gentamicin has been shown to promote PTC read-through in several genetic disorders, such as cystic fibrosis (CF), Duchenne's muscular dystrophy (DMD), hemophilia, and retinitis pigmentosa (2327). Topical gentamicin applied to the nasal epithelium of $\mathrm{CF}$ patients restored the $\mathrm{CF}$ transmembrane conductance regulator, while intravenous gentamicin has also been shown to improve the prognosis of patients with CF and DMD (28-31).

More than 800 distinct mutations have been identified in RDEB patients, in which the prevalence of nonsense mutations in RDEB approaches $30 \%(32,33)$. Nonsense mutations result in PTCs that cause a truncated or unstable type VII collagen. Therefore, nonsense suppression therapy to overcome PTCs would be of considerable benefit to the $30 \%$ of RDEB patients who harbor nonsense mutations. Using keratinocyte and fibroblast cultures from several RDEB patients harboring nonsense mutations, we showed that aminoglycosides induced PTC read-through and restored functional, full-length type VII collagen (34). In addition, we showed that aminoglycosides were capable of promoting PTC read-through and inducing fulllength type VII collagen in HEK293 cells transfected with type VII collagen expression constructs harboring 22 different known RDEB nonsense mutations. Considering these promising in vitro results, we performed a double-blind, placebo-controlled clinical trial by treating defined test sites in 5 RDEB patients harboring nonsense mutations with shortterm topical and intradermally injected gentamicin to determine whether gentamicin could induce PTC read-through and create new type VII collagen and AFs in RDEB patients with nonsense mutations. We found that gentamicin treatment delivered by either method induced new type VII collagen and AFs. The generation of new type VII collagen and AFs in the topical gentamicin-treated test sites was also associated with a trend toward improved wound closure, more durable closed wounds, and a reduction in new blister formation.

\section{Results}

Patients. Five RDEB patients with bona fide nonsense mutations were recruited from previously published studies of RDEB patients $(35,36)$. The patients were first recruited in February 2015, and the study was completed in September 2016. All 5 patients (3 adults and 2 children) met our inclusion criteria, completed the study, and were assessed for the primary and secondary end points (Figure 1). The primary end points for this double-blind and placebocontrolled pilot study were the expression of new type VII collagen and AFs in the dermal-epidermal junction (DEJ) of the test sites and an assessment of safety, especially the detection of any ototoxicity or nephrotoxicity as well as the production of anti-type VII collagen antibodies. A secondary end point was assessment of wound closure of the topically treated open wounds. For topical treatment, all 5 patients had 2 open erosive test sites that received either topical gentamicin $0.1 \%$ ointment or placebo (ointment alone) 3 times each day for 2 weeks. For intradermal treatment, in 4 of the same 5 patients (1 patient declined to participate in this

Table 1. Summary of mutations, type VII collagen expression, and AFs of the 5 study patients

$\begin{array}{lcccccc}\text { ID } & \text { PT1 } & \text { PT2 } & \text { PT3 } & \text { PT4 } & \text { PT5 } & \text { NHS } \\ \text { Sex } & \text { Male } & \text { Male } & \text { Female } & \text { Female } & \text { Male } \\ \text { Age (yr) } & 29 & 16 & 31 & 26 & 8 & \text { R613X/R1683X } \\ \text { Allele 1/allele 2 } & \text { R578X/V168Gf5X12 } & \text { R578X/R578X } & \text { R2814X/IVS17-2delA } & \text { R236X/IVS85-1G>A } & - \text { /- } \\ \text { Type VII collagen at DE) \% } & 2.0 & 0 & 4.5 & 11.6 & 0.8 & 0 \\ \text { AFs }^{B} & 0 & 0 & 0 & + & 100\end{array}$

${ }^{A}$ Assessed by IF staining using a rabbit polyclonal antibody to type VII collagen. Expression levels at the DEJ were calculated from comparison with NHS; see Methods. ${ }^{B}$ Assessed by IEM using a mouse monoclonal antibody to NC1 and scored as absent (0); thin and wispy (+); and full complement of mature AFs (+++++). NHS from multiple sources was used for comparison with patient samples. 

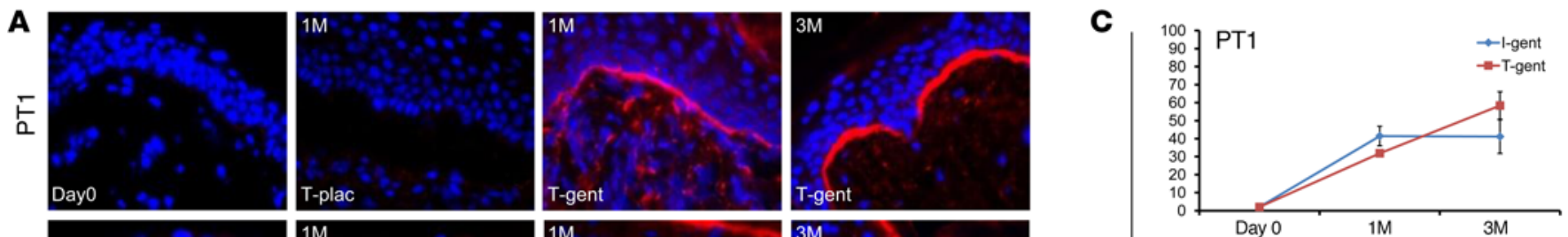

$\stackrel{5}{5}$
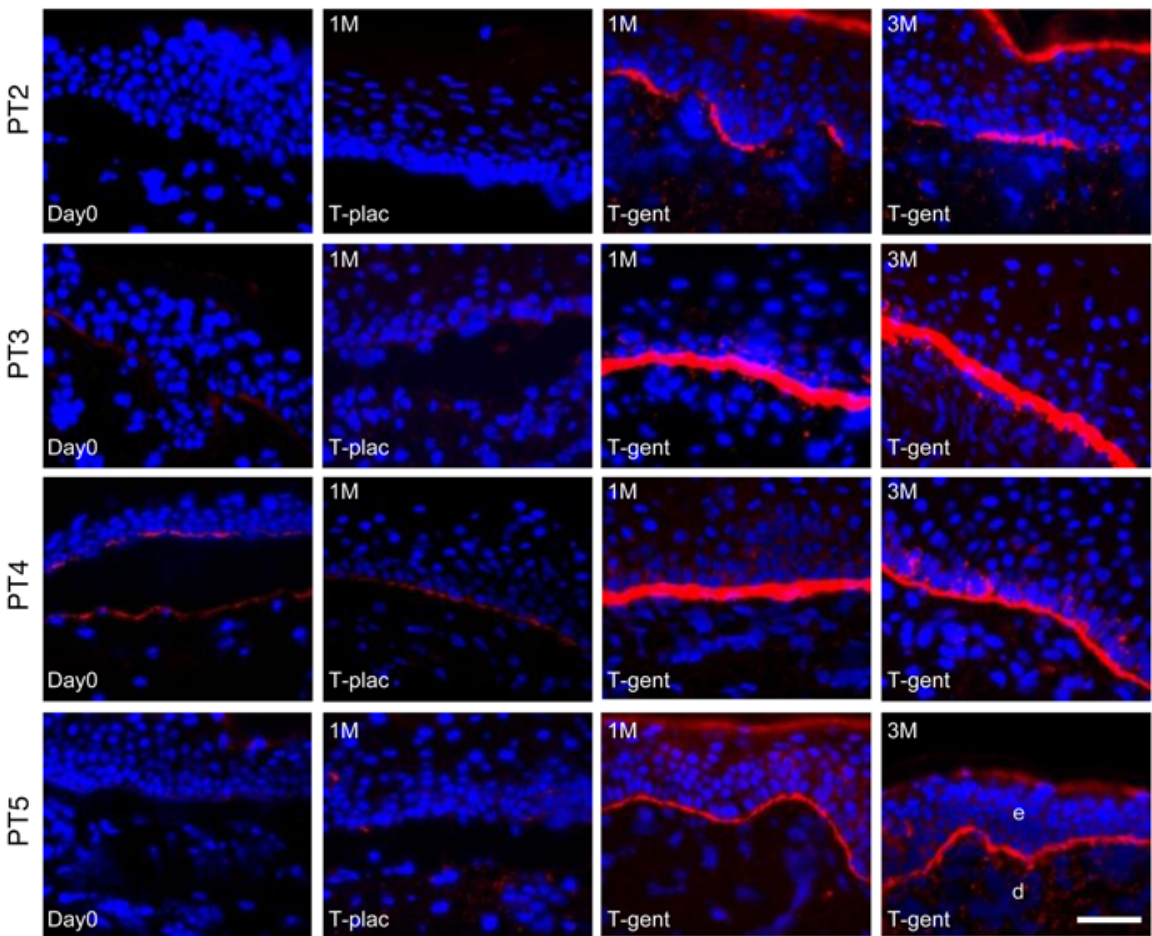

홍
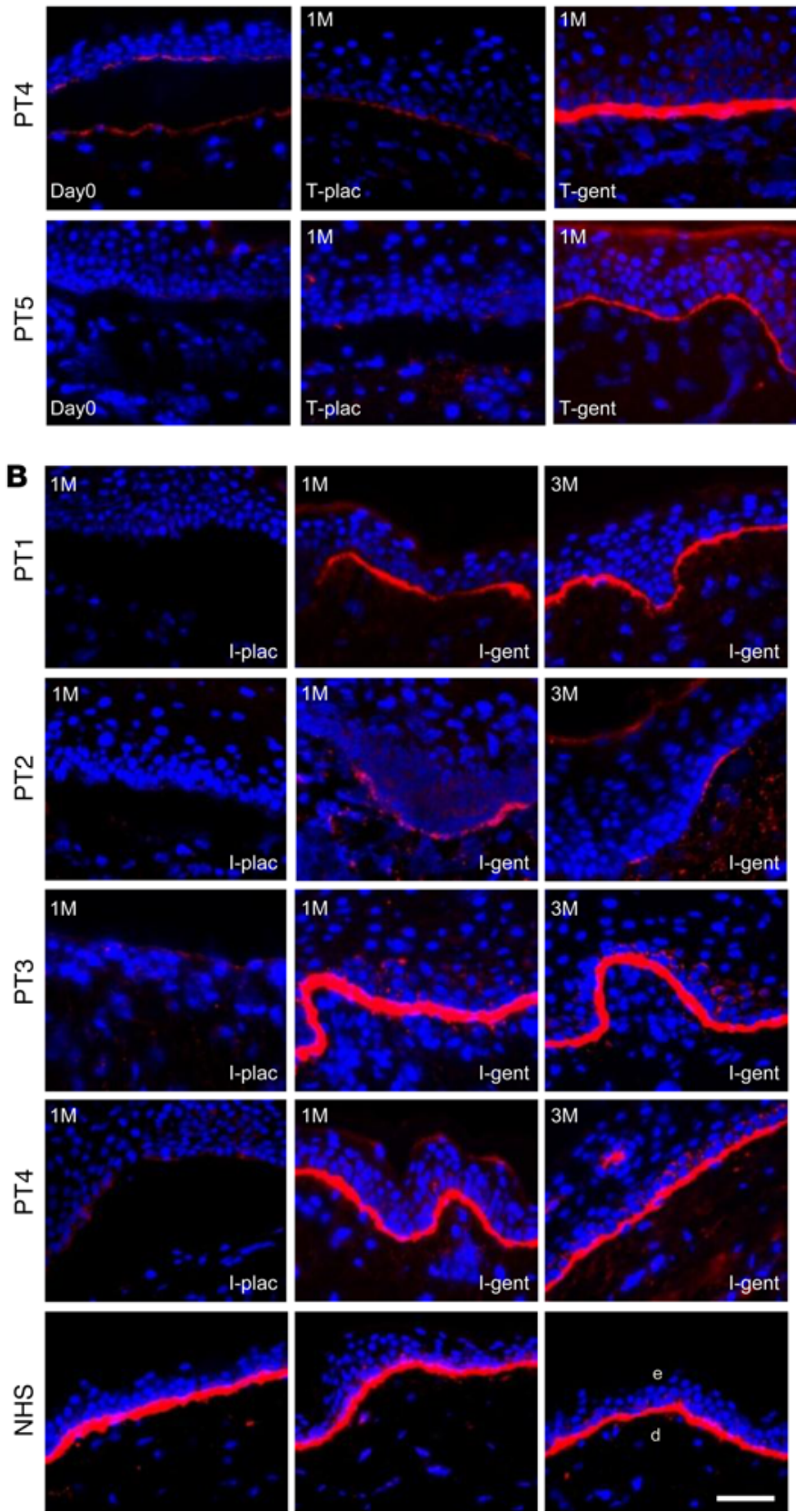
Figure 2. Topical and intradermal gentamicin treatment generated new type VII collagen in RDEB patients. IF staining with a polyclonal antibody to type VII collagen on skin biopsy specimens taken at day 0,1 month $(1 \mathrm{M})$, and 3 months $(3 \mathrm{M})$ after treatment with topical gentamicin (T-gent) or topical placebo (T-plac) (A) and intradermally injected gentamicin (I-gent) or placebo (I-plac) (B). Note that gentamin induced type VII collagen at the DEJ of the skin of all 5 patients who received topical gentamicin applied to open erosive test sites (A) and that intradermally injected gentamicin induced type VII collagen at the DEJ of all 4 patients who received the injections (B). All images were obtained using the same camera at identical exposure times. (C) Mean average fluorescence intensity for type VII collagen immunoreactivity was obtained from the images shown in panels $\mathbf{A}$ and $\mathbf{B}$. Frozen sections were probed with a polyclonal antibody to type VII collagen, and the intensity of type VII collagen at the DEJ of each specimen was measured by computer-assisted Image] software and compared with the intensity of type VII collagen in NHS. The $y$ axis shows the intensity of type VII collagen in the DEJ of the test sites expressed as a percentage of the average obtained from NHS (100\%). Data represent the mean \pm SD. (D) Mean average fluorescence for type VII collagen expression from each patient was determined and compiled for each treatment. Mean \pm SEM are shown to the right of individual sample data. $P=0.037(n=5)$ for topical and $P=0.043(n=4)$ for intradermal. Statistical significance was determined by 2-tailed Welch's $t$ test. PT1, patient 1; e, epidermis; d, dermis. Scale bars: $50 \mu \mathrm{m}$.

part of the trial), 2 intact skin test sites remote from topical sites were intradermally injected with $8 \mathrm{mg}$ of gentamicin or placebo each day for 2 days. Intradermal injections of gentamicin and placebo were not done for patient 5 due to the wishes of the patient and his parents. All 5 patients had follow-up visits at 1 month and 3 months. The clinical data of the patients participating in the study are shown in Table 1. By immunofluorescence (IF) staining with a polyclonal antibody to the $\mathrm{N}$-terminal noncollagenous domain of type VII collagen (NC1), patient 2 expressed no type VII collagen at his DEJ. The other 4 patients all had marked decreased expression of type VII collagen at their DEJ, with less than $12 \%$ of that expressed in normal human skin (NHS). AFs were evaluated by immunoelectron microscopy (IEM) with a monoclonal antibody specific for type VII collagen. As shown in Table 1,4 of the 5 patients had an absence of AFs in the skin. One of the patients had sparse, thin, wispy AFs.

Gentamicin induced new type VII collagen and AFs. We evaluated the expression of type VII collagen at the DEJ on the skin-biopsy specimens by IF for baseline biopsies and topical (Figure 2A) and intradermal (Figure 2B) gentamicin- or placebo-treated sites at months 1 and 3. Patient 1 and patient 2 had minimal to no detectable type VII collagen expression at baseline or in placebo-treated sites. In contrast, at months 1 and 3, there was increased type VII collagen expression at the DEJ of sites treated with both topical and intradermal gentamicin. Patients 3 and 4 had minimal type VII collagen at the DEJ at baseline and in placebo-treated sites. At months 1 and 3, however, the gentamicin-treated sites showed continuous, new type VII collagen at the DEJ that exceeded that of NHS. Patient 5 had minimal type VII collagen expression at baseline or in the placebo-treated sites. In contrast, there was increased type VII collagen expression at the DEJ at sites treated with topical gentamicin at both 1 and 3 months. Similar results were obtained when skin specimens were additionally probed with 2 commercially available monoclonal antibodies to the NC1 domain of type VII collagen (data not shown).
Figure 2C shows the quantification of type VII collagen expression at the DEJ relative to that of NHS (100\%). At 1 month, patients 3 and 4 had type VII collagen expression that was $153 \%$ and $166 \%$ of that in NHS, respectively. Surprisingly, the type VII collagen expression was sustained at the 3-month clinic visit and was unchanged from 1 month for patient 3 and reduced to $50 \%$ of that in NHS for patient 4. Although all 5 patients responded to gentamicin, as shown in Figure 2C, the responses were variable. Patient 1 had a baseline type VII collagen level that was only $2 \%$ of that of NHS, and topical and intradermally injected gentamicin increased the expression to $58 \%$ and $40 \%$, respectively. Patient 2 had no baseline type VII collagen expression, and at 1 month, topical and intradermal gentamicin restored the type VII collagen expression to $19 \%$ and $18 \%$, respectively. Patient 5 had a baseline expression of type VII collagen of less than $1 \%$ of that in NHS. Topical gentamicin increased the type VII collagen expression above $20 \%$ at months 1 and 3 . For all 5 patients, test sites treated with placebo did not reveal any type VII collagen expression at the DEJ above baseline.

Since the IF analysis from each patient at each time point was from 1 skin sample, to generate a statistically significant measure of type VII collagen induction in response to gentamicin treatment, we compiled type VII collagen IF data from all 5 patients 1 month after receiving either topical or intradermal gentamicin or placebo. As shown in Figure 2D, compared with placebo-treated sites, the gentamicin-treated sites exhibited a statistically significant increase in type VII collagen production $(P=0.037$ for topical and $P=0.043$ for intradermal).

To determine whether the induced type VII collagen was full length, we performed IF staining of patient skin sections at day 0 and 1 month after gentamicin treatment using a monoclonal antibody, LH24, specific to the C-terminal noncollagenous domain (NC2) of type VII collagen. Before treatment, there was no detectable full-length type VII collagen at the DEJ. In contrast, after gentamicin treatment, there was increased type VII collagen observed at the DEJ for all 5 patients, consistent with the results from IF staining with the polyclonal anti-NC1 antibody (Supplemental Figure 1; supplemental material available online with this article; https://doi.org/10.1172/JCI92707DS1). These data indicate that gentamicin-induced type VII collagen is indeed full length.

As shown in Supplemental Figure 2, the sites treated with topical gentamicin exhibited more adherence of the epidermis to the dermis compared with the placebo-treated sites. In contrast to the fluidity of type VII collagen at the DEJ in response to gentamicin, another DEJ component, laminin 332, was not altered from baseline at 1 and 3 months in any of the test sites when frozen sections were labeled with a monoclonal antibody to laminin 332 (data not shown).

To determine whether the gentamicin-induced type VII collagen could create new AFs at the DEJ, we carried out IEM using a monoclonal antibody to type VII collagen. As shown in Figure 3, there was no labeling with gold particles or AF structures observed in the test sites of patient 3 or patient 4 that were treated with placebo at 1 month. In contrast, the topical gentamicintreated test sites of patient 3 and patient 4 had abundant antitype VII collagen immunogold labeling of the lamina densa and multiple, mature, large AF structures that persisted for at least 

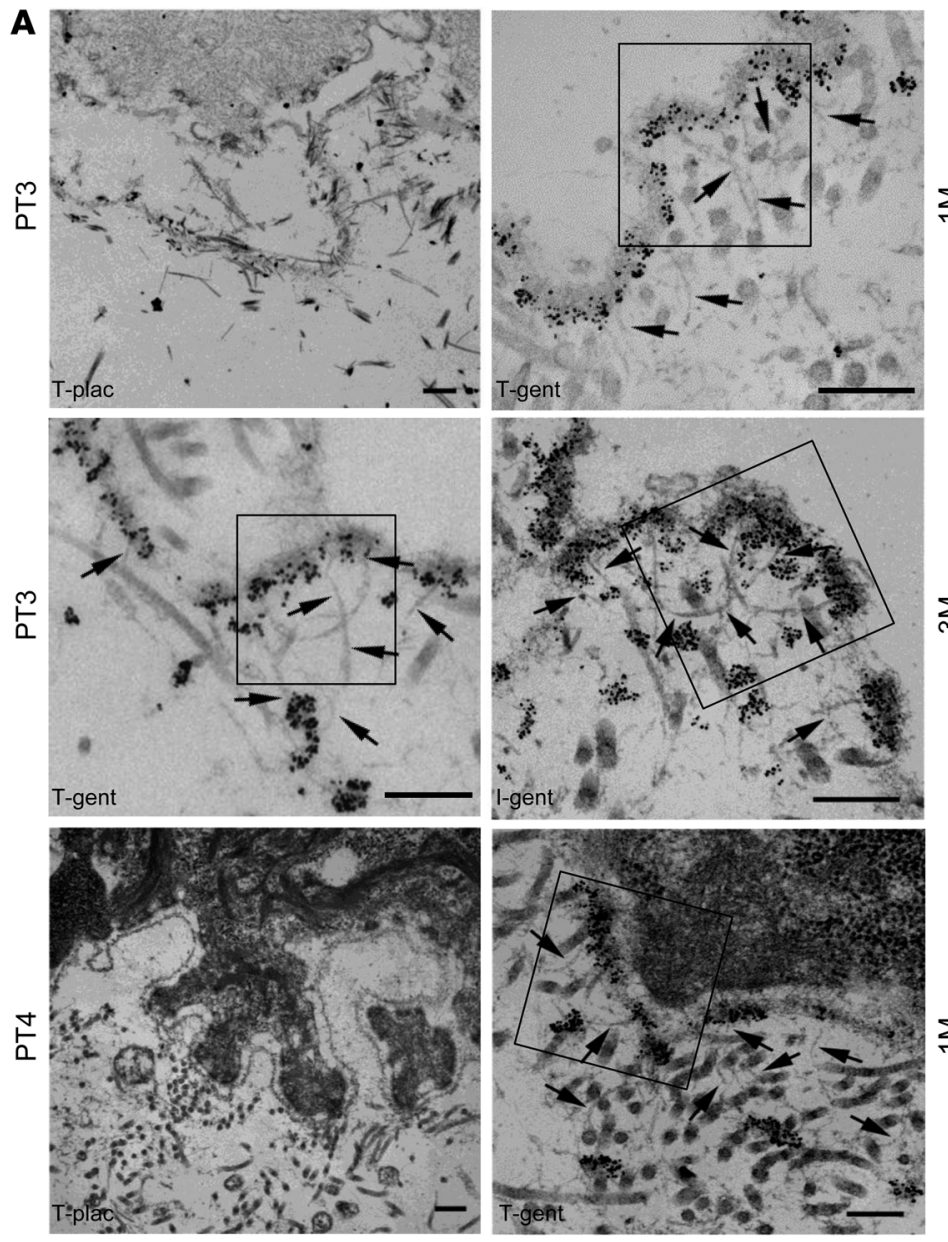

B

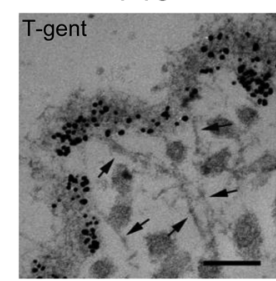

$1 \mathrm{M}$
PT3

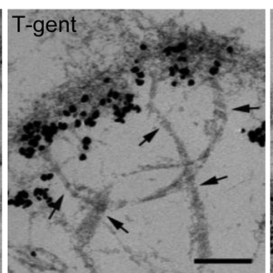

$3 \mathrm{M}$
PT3

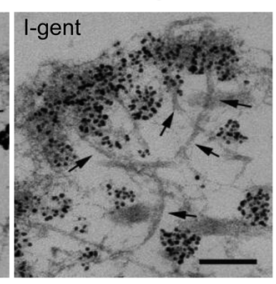

$3 \mathrm{M}$
Figure 3. Topical or intradermal gentamicin treatment generated new AFs in RDEB patients. Skin sections taken from patients 3 and 4 at 1 month and 3 months after treatment, as indicated, were subjected to IEM by labeling en bloc with a murine monoclonal antibody, mAb 185 , to type VII collagen, followed by anti-mouse IgG-conjugated immunogold particles (black dots). The photomicrographs consist of low magnification images shown in $\mathbf{A}$ with black boxes highlighting the regions shown in the high magnification images (B). Note that in the placebo-treated test sites, there was no labeling of the DEJ and there were no visible AFs in either patient. In contrast, skin biopsy samples from both topical and intradermally injected gentamicin test sites exhibited intense gold labeling of the lamina densa and many well-labeled AFs (arrows). Scale bars: $250 \mathrm{~nm}$ (A); $100 \mathrm{~nm}$ (B).

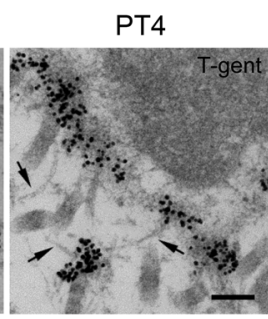

$1 \mathrm{M}$
3 months, demonstrating correction of the major ultrastructural abnormality seen in RDEB skin. Likewise, for patient 3, intradermal gentamicin also resulted in robust labeling of the lamina densa and AFs. Taken together, these data indicate that administration of gentamicin can generate new type VII collagen and new AFs in these RDEB patients.

Topical gentamicin improved wound closure and reduced blister formation. RDEB patients have severe skin fragility, and slight trauma raises new skin blisters and wounds. The patients in this study were not immobilized and were allowed their normal routine activity throughout the study. For the test sites that were treated topically with gentamicin or placebo, 2 open wounds were selected on each patient and matched for similar location, size, and chronicity (see Table 2). RDEB wounds are quite dynamic because skin fragility and trauma play large roles in the induction of new open wounds. A given wound will close and heal at one point in time and then blister and open in response to minimal trauma. The predisposition for trauma of paired test sites was not controlled and was a confounding variable of the study. Nevertheless, as shown in Table 2, test sites treated with topical gentamicin trended toward improved wound closure compared with placebo-treated sites. The 2 parameters assessed and summarized in Table 2 were the percentage of wound closure compared with the initial wound area based on image analysis of standardized photographs taken at the 1 and 3 month clinic visits and new blister formation over the course of the study based on patient diaries and weekly questionnaires, as described in Methods. Compared with the placebo-treated test sites, 4 of the 5 patients' test sites treated with gentamicin demonstrated enhanced wound closure and had less new blister formation over the course of the 3-month study. Figure 4 shows representative images of test sites treated with topical placebo or gentamicin in 4 patients. In these patients, the treatment sites that received 
Table 2. Characteristics and clinical assessment of the test sites treated topically in the $\mathbf{5}$ study patients

\begin{tabular}{|c|c|c|c|c|c|c|c|c|c|c|c|}
\hline \multirow{2}{*}{$\begin{array}{l}\text { ID } \\
\text { Treatment }\end{array}$} & & \multicolumn{2}{|c|}{ PT 1} & \multicolumn{2}{|c|}{ PT 2} & \multicolumn{2}{|c|}{ PT 3} & \multicolumn{2}{|c|}{ PT 4} & \multicolumn{2}{|c|}{ PT 5} \\
\hline & & Gentamicin & Placebo & Gentamicin & Placebo & Gentamicin & Placebo & Gentamicin & Placebo & Gentamicin & Placebo \\
\hline Wound history ${ }^{A}$ & & $>3 y r$ & $>3 \mathrm{yr}$ & $1-3 y r$ & $1-3 \mathrm{yr}$ & $>3 \mathrm{yr}$ & $>3 \mathrm{yr}$ & $1-3 \mathrm{yr}$ & $<1 \mathrm{yr}$ & $<1 \mathrm{yr}$ & $<1 \mathrm{yr}$ \\
\hline \multirow[t]{2}{*}{ Wound closure ${ }^{B}$} & $1 \mathrm{mo}$ & +++ & + & +++ & + & +++ & + & + & +++ & +++ & ++ \\
\hline & $3 \mathrm{mo}$ & +++ & +++ & +++ & + & +++ & + & + & + & +++ & ++ \\
\hline & $0-3$ mo & 1 & 3 & 1 & 3 & 1 & 4 & 1 & 1 & 0 & 2 \\
\hline
\end{tabular}

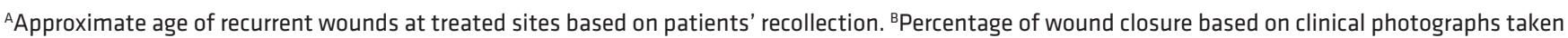
at day 0 , month 1 , and month 3; see Methods. Percentage of wound closure:,$+++ 85 \%-100 \% ;++, 50 \%-85 \% ;+,<50 \%$. ${ }^{c}$ Number of blistering events over the 1 month and 3 months of the study were recorded in patient diaries. Each new blister event observed at treatment site was counted as 1.

topical gentamicin closed within 1 month and were still intact at 3 months. In contrast, the placebo-treated wounds for these patients were more often open and continually developed new blisters and erosions throughout the course of the study.

Safety. A number of safety parameters were performed before treatment and at each posttreatment visit. These included complete blood counts, blood urea nitrogen, creatinine, calculated creatinine clearance, electrolytes, liver function tests, and pure-tone audiometry. None of these parameters changed significantly after treatment at 1 and 3 months.

Because gentamicin can induce PTC read-through and create new full-length type VII collagen, including type VII collagen domains that the patient's immune system had not seen previously, there was the possibility that the patients would generate antitype VII collagen antibodies. If they did make such antibodies, it was possible that these treated RDEB patients would then develop a secondary disease, namely, a well-described acquired autoimmune bullous disease called epidermolysis bullosa acquisita (EBA) $(37,38)$. EBA patients make pathogenic anti-type VII collagen antibodies that bind to and perturb the function of their AFs. Therefore, we determined whether gentamicin-induced type VII collagen would induce an immune response in these RDEB patients. Sera from gentamicin-treated RDEB patients were obtained at day 0 and 1 month after treatment and then subjected to tests for circulating anti-type VII collagen antibodies with a commercially purchased ELISA coated with NC1 and NC2 antigens to determine the presence of anti-type VII collagen antibodies as described (35). As shown in Figure 5A, none of the patients' sera exhibited any increases in anti-type VII collagen IgG at 1 month after gentamicin treatment. In addition, direct IF staining performed on all patients' skin at months 1 and 3 did not reveal any anti-type VII collagen antibody deposits in the DEJ of their skin (Figure $5 \mathrm{~B}$ ). We were able to obtain sera from 3 of our patients 1 year after initial treatment. With ELISA, there was no increase in anti-type VII collagen antibodies in these patients (data not shown).

\section{Discussion}

In the study, we found that both topical and intradermal administration of gentamicin to RDEB patients with nonsense mutations induced PTC read-through and created new type VII col- lagen and AFs that persisted for at least 3 months. Importantly, short-term gentamicin by either route of administration did not produce any untoward side effects in any of the patients. In addition, the open wounds treated with topical gentamicin exhibited improved epidermal-dermal adherence, reduced new blister formation, and enhanced wound closure compared with that of wounds treated with placebo.

The goal of any RDEB therapy is to generate sufficient levels of functional full-length type VII collagen that is properly located in the DEJ of the patient's skin and that creates sufficient AF structures to provide good adherence between the epidermis and dermis of the skin. One does not need to have $100 \%$ of the normal complement of AFs observed in NHS to have good epidermal-dermal adherence. Rather, there are several lines of evidence to show that only $35 \%$ of the number of AFs in NHS is sufficient to provide good epidermal-dermal adherence $(39,40)$. Therefore, restoration of type VII collagen to $35 \%$ of that seen in NHS or greater is a valid therapeutic goal. In this study, we showed that gentamicin induced PTC read-through and restored type VII collagen at the DEJ of 3 RDEB patients to levels of more than $50 \%$ that of NHS.

Our previous in vitro study utilized fibroblasts from 2 patients enrolled in the current study (patients 2 and 5) and showed that gentamicin induced type VII collagen expression at 15\%-40\% of that of normal human fibroblasts (34). In the present human study, in response to gentamicin, these 2 patients generated type VII collagen levels that were $19 \%-23 \%$ of that of NHS. Patient 3 had the greatest response to gentamicin, with type VII collagen levels exceeding that of normal skin. Patient 3's fibroblasts in culture, likewise, generated the highest levels of type VII collagen in response to gentamicin (data not shown). Similar correlations of the in vitro-cultured cell responses and in vivo clinical responses to gentamicin have been reported in patients with CF (31). In our study, the number of patients is small, but it is possible that the in vitro responses of cell cultures to gentamicin may predict the clinical response of the patient to gentamicin therapy.

In this study, it is interesting to note that despite stop codon mutations, the majority of the RDEB patients expressed small amounts of mutated type VII collagen before gentamicin treatment ( 4 of 5 patients; see Table 1). Similar findings were reported in other genetic diseases caused by PTCs, such as CF and DMD. 
A

A Day 0

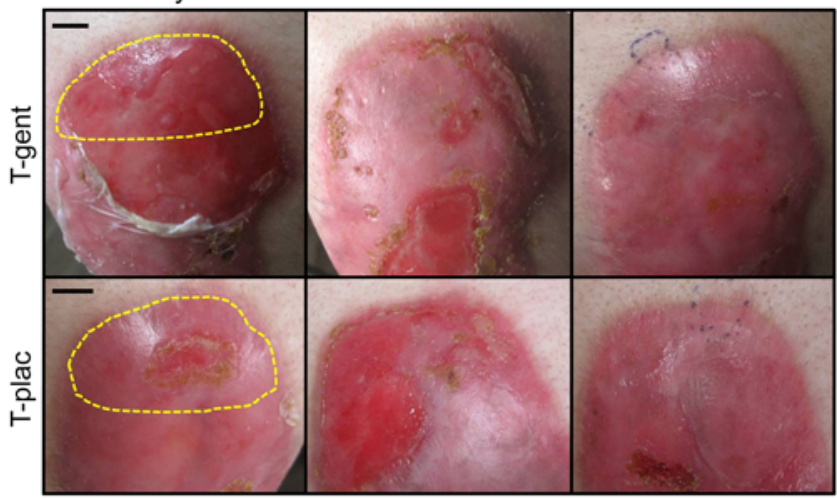

B

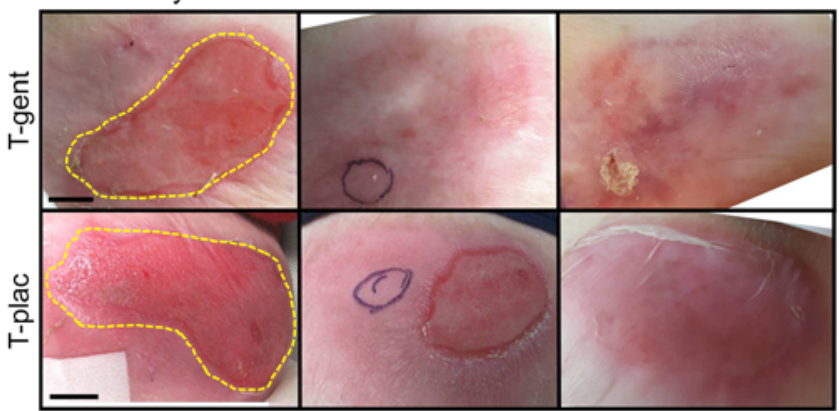

C

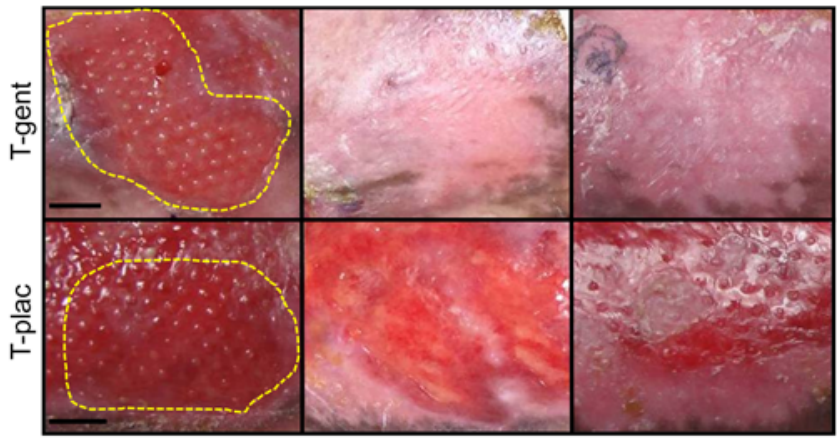

D

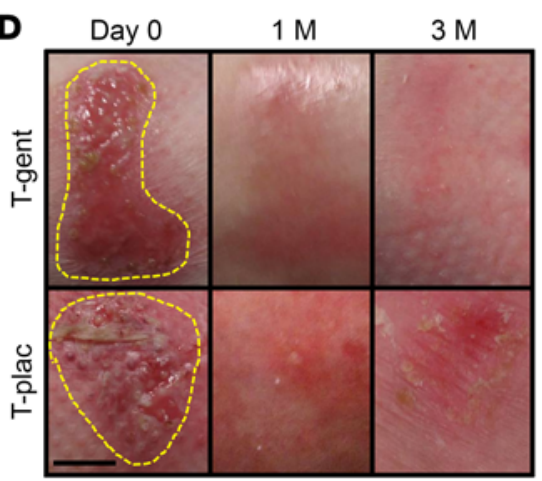

In addition, it was reported that baseline protein expression was responsible for stable transcripts escaping nonsense-mediated decay (NMD) and appeared to be predictive of a positive gentamicin response in patients with $\mathrm{CF}$ and DMD $(27,30)$. In accordance with these studies, the RDEB patients who had some baseline type VII collagen at their DEJ responded more favorably than patients who had minimal or no type VII collagen in their skin. For example,
Figure 4. Topical gentamicin improved wound closure. Representative photographs of the open erosion test sites prior to treatment and at day 0,1 month, and 3 months after treatment with either topical gentamicin or topical placebo in patient 1 (A), patient 2 (B), patient 3 (C), and patient 5 (D). Yellow dotted lines indicate the treated areas. Scale bars: $1 \mathrm{~cm}$.

patient 3 and patient 4 , who had the highest levels of pretreatment type VII collagen in their skin, responded the best to gentamicin, with induced new type VII collagen levels of over $150 \%$ of those of NHS. In contrast, gentamicin treatment of patient 2, who had no pretreatment type VII collagen, resulted in new type VII collagen expression that was only $20 \%$ of that observed in NHS. These data indicate that mRNA transcripts escaping NMD may serve as a template for stop codon read-through induced by gentamicin. As with CF and DMD, it is possible that having a basal level of type VII collagen at the DEJ of skin can potentially serve as a marker for those most likely to respond to PTC read-through treatment.

Compared with the proteins generated by gentamicin in $\mathrm{CF}$ and DMD patients with nonsense mutations, type VII collagen is a much larger protein ( $\mathrm{MW}=900 \mathrm{kDa}$ as a trimer) that becomes conformed into even larger connective tissue structures. Collagens are long-lived, stable macromolecules with slow turnover times $(41,42)$. We have shown previously that injected human recombinant type VII collagen stably incorporated into the DEJ of RDEB-like mice and persisted there for at least 3 months $(7,10)$. In this study, 3 of the treated patients maintained type VII collagen levels in excess of $50 \%$ of that in NHS for at least 3 months. Given this response to short-term gentamicin treatment and the marked stability of AFs once they are formed, we believe that in RDEB patients with nonsense mutations, gentamicin could likely be delivered as short-term, pulse therapy to maintain a sufficient number of AFs at their DEJ.

Since the insertion of a random amino acid in some readthrough product may generate full-length proteins that are not fully functional, it is important to assess whether gentamicininduced type VII collagen resulted in clinical improvement. The evidence in this study that the gentamicin-induced type VII collagen was functional in these patients is the fact that it formed normal AF structures. In addition, topically administered gentamicin, compared with placebo, improved dermal-epidermal adherence, induced durable wound closure of erosive skin wounds, and decreased new blister formation at the treated sites. A previously reported study showed that needle injection of RDEB skin and the administration of fibroblast culture medium alone generated an observable clinical improvement (43). In the current study, however, neither the placebo ointment applied topically nor the placebo injected intradermally demonstrated any positive clinical effect or generated any increase in type VII collagen or AFs at the treatment sites. It is important to point out that previous intradermal injection of fibroblast culture medium was performed in open wounds, whereas the intradermal injections of placebo in the current study were performed on intact skin, not open wounds.

In RDEB, the majority of skin erosions and wounds are colonized with ambient bacteria, and therefore, any antibiotic that reduces bacterial colonization by itself will improve wound closure. Therefore, it is possible that topical gentamicin improved wound closure due to its antimicrobial effects rather than its ability to 

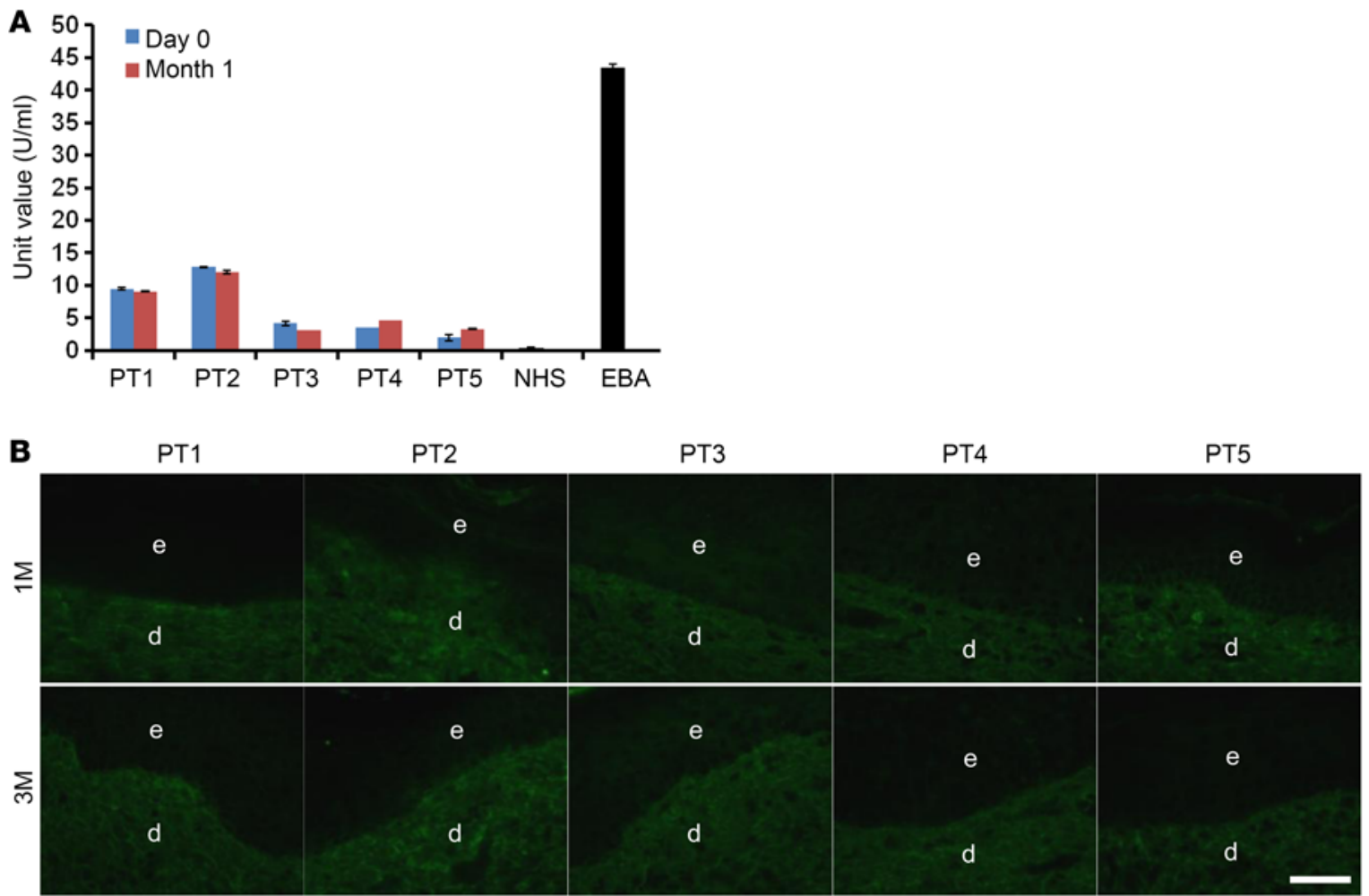

Figure 5. Topical and intradermal gentamicin do not induce anti-type VII collagen antibodies. (A) Sera were obtained from RDEB patients before and 1 month after receiving gentamicin treatments and subjected to an ELISA using a commercially available ELISA kit against type VII collagen. Note that low-level anti-type VII collagen IgG antibodies were detected in 2 of the RDEB patients at day 0 . However, none of the patients' sera exhibited any increases in anti-type VII collagen IgG antibodies at 1 month after gentamicin treatment. Serum from a patient with EBA was used as a positive control, while serum from a normal human subject (NHS) was used as a negative control. Data represent the mean \pm SD. (B) Skin biopsies from the gentamicin-treated test sites were obtained 1 month and 3 months after treatment and subjected to direct IF using an FITC-conjugated goat anti-human IgG antibody. Note that no IgG deposits of human anti-type VII collagen IgG were detected in any of the test sites. Scale bar: $50 \mu \mathrm{m}$.

induce PTC read-through and increase type VII collagen and AFs. In this study, we found that none of the test sites (either treated with gentamicin or placebo) exhibited a frank infection at day 0 and during the 3-month treatment period. While it is possible that the gentamicin-treated sites had lower bacterial counts, the generation of new type VII collagen and AFs at the DEJ only in the gentamicintreated sites strongly suggests that the likely major mechanism by which gentamicin improved clinical wound healing was by its ability to read through PTCs and generate new type VII collagen and AFs. This is consistent with our previous study showing that topical recombinant type VII collagen promotes wound closure (8).

It is well known that 2 major potential side effects associated with gentamicin use are nephrotoxicity and ototoxicity. We found no evidence of either with our short-term topical and intradermal gentamicin at 1 and 3 months after treatment. This was expected, since our topical and intradermal doses were far less than those used in previously published clinical studies (DMD and CF) in which systemic gentamicin at $7.5-10 \mathrm{mg} / \mathrm{kg}$ exhibited no toxicity $(30,31)$. The amounts of gentamicin administered in our current study were much lower than systemic doses of gentamicin routinely given to patients for infections, even if $100 \%$ of the topical or intradermal gentamicin had entered the subject's systemic circulation.

One potential side effect of newly generated type VII collagen by gentamicin treatment in these patients is the induction of autoimmunity against new type VII collagen domains that the patient's immune system had never seen. The patient's immune system may view the new type VII collagen domains as a "nonself" foreign protein and mount an immune response. Since the central deficit in RDEB patients is the lack of type VII collagen and AFs and the goal of any curative therapy is to restore type VII collagen and AFs, this potential problem will exist with any therapeutic approach to RDEB. It is important to point out that more than $60 \%$ of RDEB patients retain the NC1 domain of type VII collagen (44), which contains the most antigenic regions within type VII collagen for antibody production $(45,46)$. Therefore, most RDEB patients' immune systems have seen the most antigenic part of the molecule and recognize it as a self-protein. Fortunately, in this study, gentamicin therapy did not induce any new anti-type VII collagen autoantibodies in the sera of these patients, nor were there any new anti-type VII collagen antibody deposits in the patients' skin.

While our data demonstrated the safety and efficacy of gentamicin therapy in restoring type VII collagen to RDEB patients, there were several limitations to this pilot study related to the design and conduct of the present study. The number of patients studied was small. Only 5 patients with bona fide RDEB due to nonsense mutations in their COL7A1 gene were studied. This is because RDEB itself is exceedingly rare (0.5-1 per 1 million population) and those with nonsense mutations even rarer (47). The 
second limitation is that only 2 open-wound test sites treated with topical agents and 2 intact skin test sites treated with intradermal injections were evaluated. This limitation was due to designing a pilot study that had a tolerable number of skin biopsies for each patient. RDEB patients have been traumatized since birth by doctor visits and have a substantial fear factor, particularly involving skin biopsies. For that reason, each patient only had 4 test sites for evaluation. The small number of patients and the small number of wounds evaluated made the study less amenable to a robust statistical analysis. A third limitation was choosing the test sites. While we tried to choose open wound areas of roughly the same size and duration being open, the latter parameter relied on the patient's memory of the wounds. In general, the test sites chosen by the patients were reported to be chronic, nonhealing wounds. RDEB patients have marked skin fragility and their blisters and erosive wounds are initiated by innocuous trauma. In pairing the 2 test sites, we did not match them for how likely they were to be subjected to trauma. Therefore, it is conceivable that one test site was in an area that was more prone to trauma than its matched test site. This may have been the case with patient 4 , where the topical gentamicin-treated test site was exposed to more trauma than the placebo-treated test site. This may have confounded any woundhealing benefit provided by gentamicin despite the observed increased in type VII collagen (see Table 2). Finally, there are at least 70 reported unique nonsense mutations associated with $\mathrm{RDEB}$, and the present study only examined 5 nonsense mutations. Our data may not reflect all potential responses in patients harboring other different nonsense mutations. It is important, however, to point out that 4 nonsense mutations studied here, R236X, R578X, R1683X, and R2814X, are recurrent mutations in RDEB patients and account for $8 \%$ of total RDEB patients and $25 \%$ of RDEB patients who have nonsense mutations $(32,33)$.

While the 5 RDEB patients carry 5 different types of nonsense mutations in this study, 2 mutations, R236X and R2814, were particularly responsive to gentamicin-induced PTC read-through, while 3 other mutations, R578X, R613X and R1683X, showed less response. It has been shown previously that the type of stop codon (UGA $>\mathrm{UAG}>\mathrm{UAA}$ ) as well as the immediate downstream nucleotides $(\mathrm{C}>\mathrm{U}>\mathrm{G}>\mathrm{A})$ determine the relative read-through ability of any particular PTC mutation $(18,21)$. In our previous in vitro study of 22 RDEB nonsense mutations, we observed differential responses to gentamicin (34). However, we did not observe an apparent correlation between the read-through capacity and the type, context of each stop codon, or proximity to exon-intron boundaries, albeit with a limited sample size examined.

Various therapeutic strategies have been envisioned for RDEB based on preclinical animal models and clinical trials. These have included intradermal injection of allogeneic dermal fibroblasts or gene-corrected RDEB fibroblasts $(11,14,16)$, intradermal injection of lentiviral vectors expressing type VII collagen (48), intradermal injection or topical application of recombinant type VII collagen protein (6-8), intravenous injection of type VII collagen protein itself or fibroblasts that synthesize and secrete type VII collagen $(9,10$, 12), transplantation of bone marrow stem cells, and gene-corrected keratinocyte autografts $(13,15,17,49)$. None of the above are consistently efficacious, and some of them involve substantial risk and considerable invasiveness. Gentamicin therapy has many advan- tages compared with these therapies. First, the RDEB patients are not exposed to live cells, exogenous DNA or RNA, or viral vectors. Secondly, topical and intradermal gentamicin are commercially available, safe, inexpensive, and logistically simple to apply. Finally, gentamicin is exceedingly well characterized, with its potential side effects known, and either administration tested here can be readily performed in an outpatient clinic or at home.

In summary, our study demonstrates for what we believe is the first time that pharmacological suppression of PTC mutations by gentamicin in RDEB patients can restore sufficient levels of functional type VII collagen and AFs. The future goals of our research will be to determine the dose of gentamicin and the frequency of gentamicin delivery that optimize the generation of type VII collagen and AFs at the DEJ of RDEB patients. We will also determine whether gentamicin could be administered systemically to these patients and simultaneously treat all of their wounds, including those in the esophagus. We believe gentamicin therapy holds great promise for the $30 \%$ of RDEB patients who carry nonsense mutations. Finally, gentamicin-mediated therapy for suppressing PTCs may also be applied to other inherited skin diseases caused by nonsense mutations.

\section{Methods}

Patients and interventions. Gentamicin is a well-studied, FDA-approved antibiotic, and our study was essentially an off-label use of gentamicin. The inclusion criteria for this study were as follows: (a) RDEB patients with a nonsense mutation in COL7A1 in either 1 or 2 alleles and (b) an absence or severe decrease in type VII collagen expression at their DEJ when compared with that of NHS. The exclusion criteria for this study were as follows: (a) preexisting renal or auditory impairment, (b) allergies to aminoglycosides or sulfate compounds, (c) pregnancy, and (d) exposure to gentamicin within the past 6 weeks.

We treated 5 RDEB patients that were previously characterized with nonsense mutations $(35,36)$. All 5 patients ( 3 adults and 2 children) had severe skin disease (widespread blisters, erosions, scarring, and milia formation) and associated medical complications such as anemia and esophageal strictures. The study was double blind and placebo controlled at its onset. For the topical arm, gentamicin sulfate $0.1 \%$ ointment in white petrolatum or the ointment alone (prepared and blinded by Pasadena City Pharmacy) was applied 3 times a day to 2 open erosions for 2 weeks. Uninfected wounds were matched for size, location, and chronicity when possible (see Table 2). For the intradermal arm in the same patients, $200 \mu \mathrm{l}$ gentamicin solution (40 mg/ml, Hospira Inc.) or placebo was injected intradermally into 2 intact skin test sites, remote from the topical sites, daily for 2 days. The gentamicin test sites received 8 $\mathrm{mg}$ per day for a total dose of $16 \mathrm{mg}$. The patients had follow-up visits at 1 month and 3 months.

Clinical and safety assessments. The patients were called weekly, and a brief standardized telephone questionnaire was completed. The patients kept a daily diary and photographed their test sites once a week. Patients' test sites that received topical gentamicin or placebo were assessed at 1 month and 3 months for the percentage of wound closure and for new blister formation over the study period. For assessment of wound closure, the areas of open wounds were measured using marked, matched photographs taken during clinical visits. Standardized digital photographs were taken of the test sites, and wound 
areas were determined with an image analyzer (AlphaEase FC version 4.1.0; Alpha Innotech) as described $(8,12)$. Wound closure percentages were graded as follows: $85 \%-100 \%(+++) ; 50 \%-85 \%(++)$; and less than $50 \%(+)$. New blister formation was assessed over the 1-month and 3-month evaluation periods. Patients were asked to record new blister formation within test sites that had previously closed. Each new blister was counted as 1 .

A number of safety parameters were also assessed during initial and months 1 and 3 follow-up visits at the USC Dermatology clinic, including a complete blood count, blood urea nitrogen, creatinine, calculated creatinine clearance, electrolytes, liver function tests, and pure-tone audiometry.

Assessment of type VII collagen and AFs in the patients' skin. Prior to the onset of the study, baseline levels of type VII collagen and AFs in the DEJ of each patient were assessed at day 0 . At sites of intact skin, 8 -mm punch biopsies were obtained, divided into 2 parts - one part for quantitative IF staining for the expression of type VII collagen at the DEJ and for standard histology and a second part for IEM to assess AF structures at the DEJ, as previously described (6-13). At 1- and 3-month follow-up visits, biopsies from the test sites were obtained and processed in an identical fashion. For the IF staining, a minimum of 60 vertical frozen sections through the entire specimen were prepared and labeled with a polyclonal anti-type VII collagen antibody as described previously (6-13). Additionally, IF staining was performed on at least 20 sections with 2 different monoclonal anti-type VII collagen antibodies (clone LH7.2; Sigma-Aldrich; mAb 185, clone 32; Millipore) as well as a mouse monoclonal antibody to NC2 of type VII collagen (49). Immunolabeled vertical sections of NHS (positive control) and the experimental test sites (gentamicin treated and placebo treated) were photographed using the same camera and identical exposure times. Quantitation of type VII collagen expression by mean fluorescence intensity at the DEJ was performed by computerassisted image analysis using Image $(\mathrm{NIH})$. For each tissue sample, 6 random images from positively stained areas were taken for mean fluorescence intensity. For each image, 6 measurements were taken at $20-\mu \mathrm{m}$ intervals along the DEJ using a $20 \mu \mathrm{m} \times 20 \mu \mathrm{m}$ area. Mean averages and SEM were calculated for each sample. NHS from 3 different subjects was used as a positive control, and the expression of type VII collagen in the test sites was compared with the expression observed in NHS (100\%).

For IEM, samples were incubated with a monoclonal antibody against human type VII collagen (mAb 185, clone 32; Millipore) and evaluated as previously described (50).

Assessment of anti-type VII collagen autoantibodies. The production of serum circulating anti-type VII collagen antibodies was evaluated by ELISA following the manufacturer's procedures (MBL), as described previously (35). To evaluate whether there were any anti-type VII col- lagen antibodies deposited in the patients' skin, skin biopsy samples were subjected to direct IF staining using FITC-conjugated goat antihuman IgG (Sigma-Aldrich), as previously described $(35,37,38)$.

Statistics. To obtain statistical significance concerning the type VII collagen induction in response to gentamicin treatment, we compiled mean average fluorescence from 6 independent evaluations for type VII collagen expression from each patient biopsy at 1 month after receiving either topical or intradermal gentamicin or placebo and determined statistical significance using 2-tailed Welch's (unequal variance) $t$ test. $n=5$ for topical treatments. $n=4$ for intradermal treatments. A $P$ value of less than 0.05 was considered significant.

Study approval. The study protocol was approved by the IRB of USC, and all investigations were conducted according to Declaration of Helsinki principles. Written, informed consent was obtained from all patients prior to the onset of the study. The study was registered at ClinicalTrials.gov (NCTO2698735).

\section{Author contributions}

DTW, JC, YH, CL, and MC undertook the study and contributed to acquisition, analysis, and interpretation of the data. DK contributed to IEM. MPM was the source of the anti-NC2 antibody and contributed to the IF analysis. DTW and MC designed the clinical trial, reviewed outcomes, provided critical intellectual input, and wrote the manuscript. DTW and MC directed the study overall. All authors were involved in reviewing the manuscript and approving of the final version for submission

\section{Acknowledgments}

This work was supported in part by grants from the Epidermolysis Bullosa Research Partnership and the Epidermolysis Bullosa Medical Research Foundation (to MC and DTW) and the NIH (RC4AR060535, to MC and DTW), a VA Merit Award (to DTW), and grants from the Office of Research and Development (Palo Alto VA Medical Center to MPM) and from the NIH (R01 AR055914 to MPM). We thank the patients for granting permission to publish this information. We thank Anita Jeredjian from Pasadena City Pharmacy for formulating and blinding the topical compounds and Sara Tufa for technical support of IEM. We gratefully acknowledge Carlos Beltran and Brenda Cornejo at the USC Department of Dermatology.

Address correspondence to: David T. Woodley or Mei Chen, The USC Laboratories for Investigative Dermatology, USC/ Norris Cancer Center, Ezralow Tower, Room 6322, 1441 Eastlake Avenue, Los Angeles, California 90033, USA. Phone: 323.865.0621; Email: dwoodley@med.usc.edu (D.T. Woodley); chenm@usc.edu (M. Chen).
1. Lin AN, Carter DM. Epidermolysis bullosa: basic and clinical aspects. New York, NY: Springer-Verlag; 1992.

2. Uitto J, Christiano AM. Molecular basis for the dystrophic forms of epidermolysis bullosa: mutations in the type VII collagen gene. Arch Dermatol Res. 1994;287(1):16-22.

3. Sakai LY, Keene DR, Morris NP, Burgeson $\mathrm{RE}$. Type VII collagen is a major structural component of anchoring fibrils. J Cell Biol. 1986;103(4):1577-1586.

4. Fine JD, Johnson LB, Weiner M, Li KP, Suchindran C. Epidermolysis bullosa and the risk of life-threatening cancers: the National EB Registry experience, 1986-2006. J Am Acad Dermatol. 2009;60(2):203-211.

5. Fine JD, Hinter H. Life with epidermolysis bullosa (EB): etiology, diagnosis, multidisciplinary care and therapy. New York, NY: Springer-Verlag; 2009

6. Woodley DT, et al. Injection of recombinant

human type VII collagen restores collagen function in dystrophic epidermolysis bullosa. Nat Med. 2004;10(7):693-695.

7. Remington J, et al. Injection of recombinant human type VII collagen corrects the disease phenotype in a murine model of dystrophic epidermolysis bullosa. Mol Ther. 
2009;17(1):26-33.

8. Wang $X$, et al. Topical application of recombinant type VII collagen incorporates into the dermalepidermal junction and promotes wound closure. Mol Ther. 2013;21(7):1335-1344.

9. Woodley DT, et al. Intravenously injected recombinant human type VII collagen homes to skin wounds and restores skin integrity of dystrophic epidermolysis bullosa. J Invest Dermatol. 2013;133(7):1910-1913.

10. Hou Y, et al. Intravenously administered recombinant human type VII collagen derived from Chinese hamster ovary cells reverses the disease phenotype in recessive dystrophic epidermolysis bullosa mice. J Invest Dermatol. 2015;135(12):3060-3067.

11. Woodley DT, et al. Normal and gene-corrected dystrophic epidermolysis bullosa fibroblasts alone can produce type VII collagen at the basement membrane zone. J Invest Dermatol. 2003;121(5):1021-1028.

12. Woodley DT, et al. Intravenously injected human fibroblasts home to skin wounds, deliver type VII collagen, and promote wound healing. Mol Ther. 2007;15(3):628-635.

13. Chen M, et al. Restoration of type VII collagen expression and function in dystrophic epidermolysis bullosa. Nat Genet. 2002;32(4):670-675.

14. Ortiz-Urda S, Lin Q, Green CL, Keene DR, Marinkovich MP, Khavari PA. Injection of genetically engineered fibroblasts corrects regenerated human epidermolysis bullosa skin tissue. J Clin Invest. 2003;111(2):251-255.

15. Ortiz-Urda S, et al. Stable nonviral genetic correction of inherited human skin disease. Nat Med. 2002;8(10):1166-1170.

16. Wong $\mathrm{T}$, et al. Potential of fibroblast cell therapy for recessive dystrophic epidermolysis bullosa. J Invest Dermatol. 2008;128(9):2179-2189.

17. Wagner JE, et al. Bone marrow transplantation for recessive dystrophic epidermolysis bullosa. N Engl J Med. 2010;363(7):629-639.

18. Zingman LV, Park S, Olson TM, Alekseev AE, Terzic A. Aminoglycoside-induced translational read-through in disease: overcoming nonsense mutations by pharmacogenetic therapy. Clin Pharmacol Ther. 2007;81(1):99-103.

19. Linde L, Kerem B. Introducing sense into nonsense in treatments of human genetic diseases. Trends Genet. 2008;24(11):552-563.

20. Bidou L, Allamand V, Rousset JP, Namy O. Sense from nonsense: therapies for premature stop codon diseases. Trends Mol Med. 2012;18(11):679-688.

21. Keeling KM, Xue X, Gunn G, Bedwell DM. Therapeutics based on stop codon readthrough. Annu Rev Genomics Hum Genet. 2014;15:371-394.

22. Shi M, et al. Premature termination codons are recognized in the nucleus in a reading-frame dependent manner. Cell Discov. 2015;1.

23. Barton-Davis ER, Cordier L, Shoturma DI, Leland SE, Sweeney HL. Aminoglycoside antibiotics restore dystrophin function to skeletal muscles of mdx mice. JClin Invest. 1999;104(4):375-381.

24. Bedwell DM, et al. Suppression of a CFTR premature stop mutation in a bronchial epithelial cell line. Nat Med.1997;3(11):1280-1284.

25. Wagner KR, et al. Gentamicin treatment of Duchenne and Becker muscular dystrophy due to nonsense mutations. Ann Neurol. 2001;49(6):706-711.

26. Kuschal C, DiGiovanna JJ, Khan SG, Gatti RA, Kraemer KH. Repair of UV photolesions in xeroderma pigmentosum group $\mathrm{C}$ cells induced by translational readthrough of premature termination codons. Proc Natl Acad Sci U S A. 2013;110(48):19483-19488.

27. Linde L, et al. Nonsense-mediated mRNA decay affects nonsense transcript levels and governs response of cystic fibrosis patients to gentamicin. JClin Invest. 2007;117(3):683-692.

28. Wilschanski M, et al. A pilot study of the effect of gentamicin on nasal potential difference measurements in cystic fibrosis patients carrying stop mutations. Am J Respir Crit Care Med. 2000; 161(3 Pt 1):860-865.

29. Wilschanski M, et al. Gentamicin-induced correction of CFTR function in patients with cystic fibrosis and CFTR stop mutations. N Engl JMed. 2003;349(15):1433-1441.

30. Malik V, et al. Gentamicin-induced readthrough of stop codons in Duchenne muscular dystrophy. Ann Neurol. 2010;67(6):771-780.

31. Sermet-Gaudelus I, et al. In vitro prediction of stop-codon suppression by intravenous gentamicin in patients with cystic fibrosis: a pilot study. BMC Med. 2007;5:5.

32. Wertheim-Tysarowska K, et al. The COL7A1 mutation database. Hum Mutat. 2012;33(2):327-331.

33. van den Akker PC, et al. The international dystrophic epidermolysis bullosa patient registry: an online database of dystrophic epidermolysis bullosa patients and their COL7A1 mutations. Hum Mutat. 2011;32(10):1100-1107.

34. Cogan J, et al. Aminoglycosides restore fulllength type VII collagen by overcoming premature termination codons: therapeutic implications for dystrophic epidermolysis bullosa. Mol Ther. 2014;22(10):1741-1752.

35. Woodley DT, et al. De novo anti-type VII collagen antibodies in patients with recessive dystrophic epidermolysis bullosa. J Invest Dermatol. 2014;134(4):1138-1140.

36. Gorell ES, Nguyen N, Siprashvili Z, Marinkovich MP, Lane AT. Characterization of patients with dystrophic epidermolysis bullosa for collagen VII therapy. Br JDermatol. 2015;173(3):821-823.

37. Woodley DT, Burgeson RE, Lunstrum G,
Bruckner-Tuderman L, Reese MJ, Briggaman RA. Epidermolysis bullosa acquisita antigen is the globular carboxyl terminus of type VII procollagen. J Clin Invest. 1988;81(3):683-687.

38. Woodley DT, Briggaman RA, O'Keefe EJ, Inman AO, Queen LL, Gammon WR. Identification of the skin basement-membrane autoantigen in epidermolysis bullosa acquisita. $N$ EnglJMed. 1984;310(16):1007-1013.

39. Tidman MJ, Eady RA. Evaluation of anchoring fibrils and other components of the dermalepidermal junction in dystrophic epidermolysis bullosa by a quantitative ultrastructural technique. J Invest Dermatol. 1985;84(5):374-377.

40. Kern JS, et al. Mechanisms of fibroblast cell therapy for dystrophic epidermolysis bullosa: high stability of collagen VII favors long-term skin integrity. Mol Ther. 2009;17(9):1605-1615.

41. Pelkonen R, Kivirikko KI. Hydroxyprolinemia: an apparently harmless familial metabolic disorder. N Engl J Med.1970;283(9):451-456.

42. Burgeson RE. Type VII collagen, anchoring fibrils, and epidermolysis bullosa. J Invest Dermatol.1993;101(3):252-255.

43. Venugopal SS, et al. A phase II randomized vehiclecontrolled trial of intradermal allogeneic fibroblasts for recessive dystrophic epidermolysis bullosa. J Am Acad Dermatol. 2013;69(6):898-908.e7.

44. Ortiz-Urda S, et al. Type VII collagen is required for Ras-driven human epidermal tumorigenesis. Science. 2005;307(5716):1773-1776.

45. Jones DA, Hunt SW, Prisayanh PS, Briggaman RA, Gammon WR. Immunodominant autoepitopes of type VII collagen are short, paired peptide sequences within the fibronectin type III homology region of the noncollagenous (NC1) domain. J Invest Dermatol. 1995;104(2):231-235.

46. Lapiere JC, et al. Epitope mapping of type VII collagen. Identification of discrete peptide sequences recognized by sera from patients with acquired epidermolysis bullosa. J Clin Invest. 1993;92(4):1831-1839.

47. Pfendner E, Uitto J, Fine JD. Epidermolysis bullosa carrier frequencies in the US population. JInvest Dermatol. 2001;116(3):483-484.

48. Woodley DT, et al. Intradermal injection of lentiviral vectors corrects regenerated human dystrophic epidermolysis bullosa skin tissue in vivo. Mol Ther. 2004;10(2):318-326.

49. Siprashvili Z, et al. Safety and wound outcomes following genetically corrected autologous epidermal grafts in patients with recessive dystrophic epidermolysis bullosa. JAMA. 2016;316(17):1808-1817.

50. Meneguzzi G, Marinkovich MP, Aberdam D, Pisani A, Burgeson R, Ortonne JP. Kalinin is abnormally expressed in epithelial basement membranes of Herlitz's junctional epidermolysis bullosa patients. Exp Dermatol.1992;1(5):221-229. 